\title{
Multiresolution Analysis Applied to the Monge-Kantorovich Problem
}

\author{
Armando Sánchez-Nungaray ${ }^{D}$, ${ }^{1}$ Carlos González-Flores, ${ }^{2}$ and Raquiel R. López-Martínez ${ }^{1}$ \\ ${ }^{1}$ Facultad de Matemáticas, Universidad Veracruzana, Xalapa, VER, Mexico \\ ${ }^{2}$ Escuela Superior de Ingeniera Mecánica y Eléctrica, Instituto Politécnico Nacional, Mexico City, Mexico \\ Correspondence should be addressed to Armando Sánchez-Nungaray; armsanchez@uv.mx
}

Received 17 February 2018; Accepted 4 April 2018; Published 3 June 2018

Academic Editor: Turgut Öziş

Copyright (c) 2018 Armando Sánchez-Nungaray et al. This is an open access article distributed under the Creative Commons Attribution License, which permits unrestricted use, distribution, and reproduction in any medium, provided the original work is properly cited.

\begin{abstract}
We give a scheme of approximation of the MK problem based on the symmetries of the underlying spaces. We take a Haar type MRA constructed according to the geometry of our spaces. Thus, applying the Haar type MRA based on symmetries to the MK problem, we obtain a sequence of transportation problem that approximates the original MK problem for each of MRA. Moreover, the optimal solutions of each level solution converge in the weak sense to the optimal solution of original problem.
\end{abstract}

\section{Introduction}

The optimal transport problem was first formulated by Monge in 1781 and concerned finding the optimal way in the sense of minimal transportation cost of moving a pile of soil from one site to another. This problem was given a modern formulation in the work of Kantorovich in 1942 and so is now known as the Monge-Kantorovich problem.

On the other hand, a big advantage over schemes of approximation was given in the seminal article [1]; it introduced approximation schemes for infinite linear program; in particular, it showed that under suitable assumptions the program's optimum value can be finite-dimensional linear programs and that, in addition, every accumulation point of a sequence of optimal solutions for the approximating programs is an optimal solution for the original problem. An example given in this article is the Monge-Kantorovich mass transfer $(\mathrm{MK})$ problem on the space itself, where the underlying space is compact.

In [2], a general method of approximation for the MK problem is given, where $X$ and $Y$ are Polish spaces; however, this method is noneasier implementation. Later, in [3], a scheme of approximation of MK problem is provided, which consists in giving a sequence of finite transportation problems underlying original MK problem (the space is compact); nevertheless, a general procedure is given, but the examples are in a two-dimensional cube and use the dyadic partition of the cube for approximation. Our objective is to give other families to approximate this kind of problems, based on Haar type multiresolution analysis (MRA). The advantage of using this technique is that we select a multiresolution analysis that is constructed according to the symmetries of the underlying space. Therefore, the new schemes of approximation take a lot of characteristics of the space into consideration. Note that the dyadic partition of the cube is a particular case of the MRA type Haar using translations and dilations of the underlying space.

The MRA is an important method to approximate functions in different context (signal processing, differential equations, etc.). In particular, we focus on Haar type MRA on $\mathbb{R}^{n}$; the constructions of this kind of MRA are associated with the symmetries of the spaces; thus the approximations are related to the geometrical properties of the space. In this construction, the symmetries that we use are general dilations, rotations, reflections, translations, and so forth; for more details, see [4-6].

The main objective of this paper is giving a scheme of approximation of the MK problem based on the symmetries of the underlying spaces. We take a Haar type MRA constructed according to the geometry of our spaces. Thus, 
applying the Haar type MRA based on symmetries to the MK problem, we obtain a sequence of transportation problems that approximate the original MK problem for each level of MRA. Moreover, the optimal solutions of each level solution converge in the weak sense to the optimal solution of original problem.

This paper is organized as follows. In Section 2, we introduce the basic elements of the Haar type multiresolution analysis and we give some examples of this kind of MRA. In Section 3, we present the approximation of probability measures using Haar type MRA. In Section 5, we apply the Haar type multiresolution analysis to MK problem for each level of this MRA; thus for each level, this new problem is equivalent to transport problem. Moreover, we prove that the optimal solution of MK problems is equal to the limit of the optimal solutions of underlying transportation problems when the level of the MRA goes to infinity. Finally, we give an illustrative example of this method.

\section{Haar Type Multiresolution Analysis}

We introduce the basic concepts of Haar type multiresolution analysis, following Gröchenig and Madych in [4] and Guo et al. in [5]. Similar results have been obtained independently by Krishtal et al. to be published in [6].

Let $\Gamma$ be a lattice such that $\Gamma=M \mathbb{Z}^{n}$ for any $M \in G L_{n}(\mathbb{R})$. The classical multiresolution analysis (MRA) associated with a sequence of dilations $\left\{a^{j}\right\}_{j \in \mathbb{Z}}=A$, where $|\operatorname{det} a| \leq 1$, is a sequence $\left\{V_{j}\right\}_{j \in \mathbb{Z}}$ of closed subspaces of $\mathscr{L}^{2}\left(\mathbb{R}^{n}\right)$, which satisfies the following conditions:

(i) $V_{k} \subset V_{k+1}$.

(ii) $\overline{\bigcup_{j \in \mathbb{Z}} V_{j}}=\mathscr{L}^{2}\left(\mathbb{R}^{n}\right)$.

(iii) $\bigcap_{j \in \mathbb{Z}} V_{j}=\{0\}$.

(iv) $V_{j}=D_{a^{-j}} V_{0}$.

(v) There exists $\varphi \in V_{0}$ such that $\left\{T_{\gamma} \varphi\right\}, \gamma \in \Gamma$, is an orthonormal basis for $V_{0}$.

Let $B$ be a finite subgroup of $G L_{n}(\mathbb{R})$ such that $|\operatorname{det} b|=1$ for all $b \in B$ and $\Gamma=B(\Gamma)$. The operator generator by the dilations $D_{b}, b \in B$, and the translations $T_{\gamma}, \gamma \in \Gamma$ form a group. The relation

$$
\left(D_{c} T_{\tau}\right)\left(D_{b} T_{\gamma}\right) f=D_{c b} T_{b^{-1} \tau+\gamma} f .
$$

allows us to define the operation to $B \times \Gamma$ given by

$$
(c, \tau) \cdot(b, \gamma)=\left(c b, b^{-1} \tau+\gamma\right)
$$

and we obtain a new group denoted by $B \Gamma$. The $B \Gamma$-invariant spaces are the closed subspaces $V$ of $\mathscr{L}^{2}\left(\mathbb{R}^{n}\right)$ such that $D_{b} T_{\gamma} f \in V$ whenever $f \in V, b \in B$, and $\gamma \in \Gamma$. This leads us to the following version of $(\mathrm{v})$ :

$\left(\mathrm{v}^{\prime}\right)$ There exists $\varphi \in V_{0}$ such that $\left\{D_{b} T_{\gamma} \varphi\right\}, b \in B$ and $\gamma \in \Gamma$, is an orthonormal basis for $V_{0}$.

In consequence, we have the following concept.
Definition 1. Let $A=\left\{a^{j}\right\}_{i \in \mathbb{Z}}$ be dilatation set, let $B$ be a finite subgroup of $G L_{n}(\mathbb{R})$ with $|\operatorname{det} b|=1$, and let $\Gamma$ be a complete lattice such that $\Gamma=B(\Gamma)$. The multiresolution analysis associated with the dilation set $A$ and the group $B$ or $A B$-MRA is a collection $\left\{V_{j}\right\}_{j \in \mathbb{Z}}$ of closed subspaces of $\mathscr{L}^{2}\left(\mathbb{R}^{n}\right)$, which satisfies conditions (i), (ii), (iii), (iv), and ( $\left.{ }^{\prime}\right)$.

The classical MRA is considered as an $A B$-MRA when $B$ is the trivial group. Note that the space $V_{0}$ is not generated by the $\Gamma$-translations of the single scaling function $\varphi$; however, the relation $D_{b} T_{\gamma} \varphi=T_{b \gamma} D_{b} \varphi$ and the conditions $B(\Gamma)=\Gamma$ imply that the functions $D_{b} \varphi$, with $b \in B$, are the generators of $V_{0}$. Also, we have the following set:

$$
\Gamma^{j}=\left\{a^{j} \gamma: \gamma \in \gamma\right\}
$$

Note that $\Gamma^{j} \subset \Gamma^{j+1}$ and $B \Gamma^{j}=\Gamma$.

We consider the scaling function $\varphi=E \chi_{\Delta}$, where $\chi_{\Delta}$ is the characteristic function of $\Delta \subset \mathbb{R}^{n}, E \in \mathbb{R}$, and $\|\varphi\|_{2}=1$. The region $\Delta$ satisfies

$$
\bigcup_{\alpha \in B \Gamma} \Delta_{\alpha}=\mathbb{R}^{n}
$$

where int $\Delta_{\alpha} \cap$ int $\Delta_{\alpha^{\prime}}=\emptyset$ for $\alpha \neq \alpha^{\prime}$ and $\Delta_{\alpha}$ is the action of $\alpha=(b, \gamma)$ on $\Delta$. In addition, the symbol $\Delta_{\alpha}^{j}$ denotes the translation and scaling of the region $\Delta$ by $\alpha$ and $a^{j}$, respectively. Thus, the function $D_{a^{j}} D_{b} T_{\gamma} \varphi=|\operatorname{det} a|^{-j / 2} E \chi_{\Delta_{\alpha}^{j}}$ is denoted by $\varphi_{\alpha}^{j}$. And so we have the following relation:

$$
\sum_{\alpha \in B \Gamma} \varphi_{\alpha}^{j}=|\operatorname{det} a|^{-j / 2} E \sum_{\alpha \in B \Gamma} \chi_{\Delta_{\alpha}^{j}}=|\operatorname{det} a|^{-j / 2} E \cdot \chi_{\mathbb{R}^{n}} .
$$

Finally, we define $P^{j}$ as the orthogonal projection from $\mathscr{L}^{2}\left(\mathbb{R}^{n}\right)$ to $V_{j}$ which is given by

$$
P_{j} f=\sum_{\alpha \in B \Gamma}\left\langle f, \varphi_{\alpha}^{j}\right\rangle \varphi_{\alpha}^{j}
$$

for all $f \in \mathscr{L}^{2}\left(\mathbb{R}^{n}\right)$.

We show some examples of the multiresolution analysis associated with the dilation set $A$ and the finite group $B$ :

(1) We take a matrix $a=q=\left(\begin{array}{cc}1 & -1 \\ 1 & 1\end{array}\right)$ and the group $B=$ $\left\{b_{i}\right\}, 0 \leq i \leq 7$, of symmetries of unit square; thus,

$$
\begin{aligned}
& b_{0}=\left(\begin{array}{ll}
1 & 0 \\
0 & 1
\end{array}\right), \\
& b_{1}=\left(\begin{array}{ll}
0 & 1 \\
1 & 0
\end{array}\right), \\
& b_{2}=\left(\begin{array}{cc}
0 & -1 \\
1 & 0
\end{array}\right), \\
& b_{3}=\left(\begin{array}{ll}
-1 & 0 \\
0 & 1
\end{array}\right),
\end{aligned}
$$



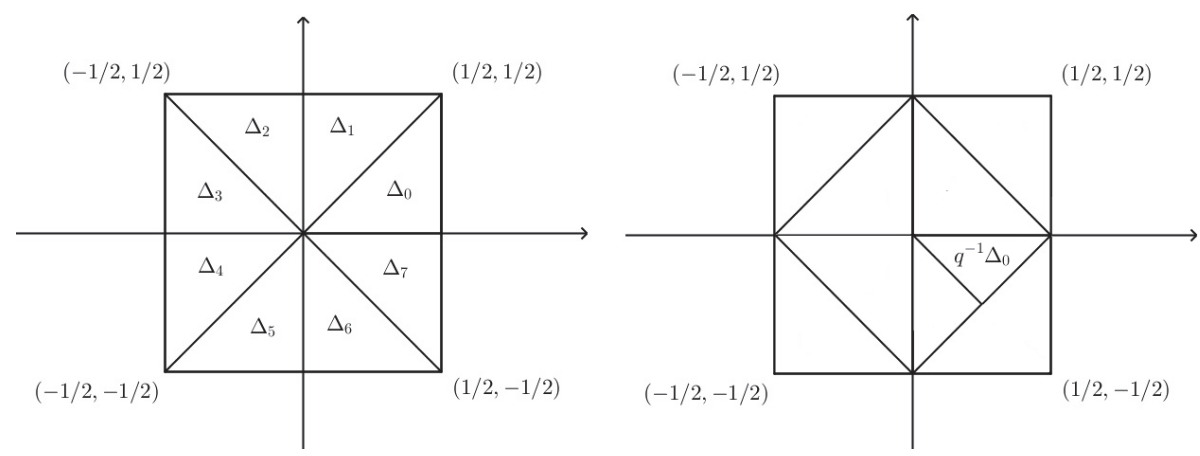

FiguRe 1
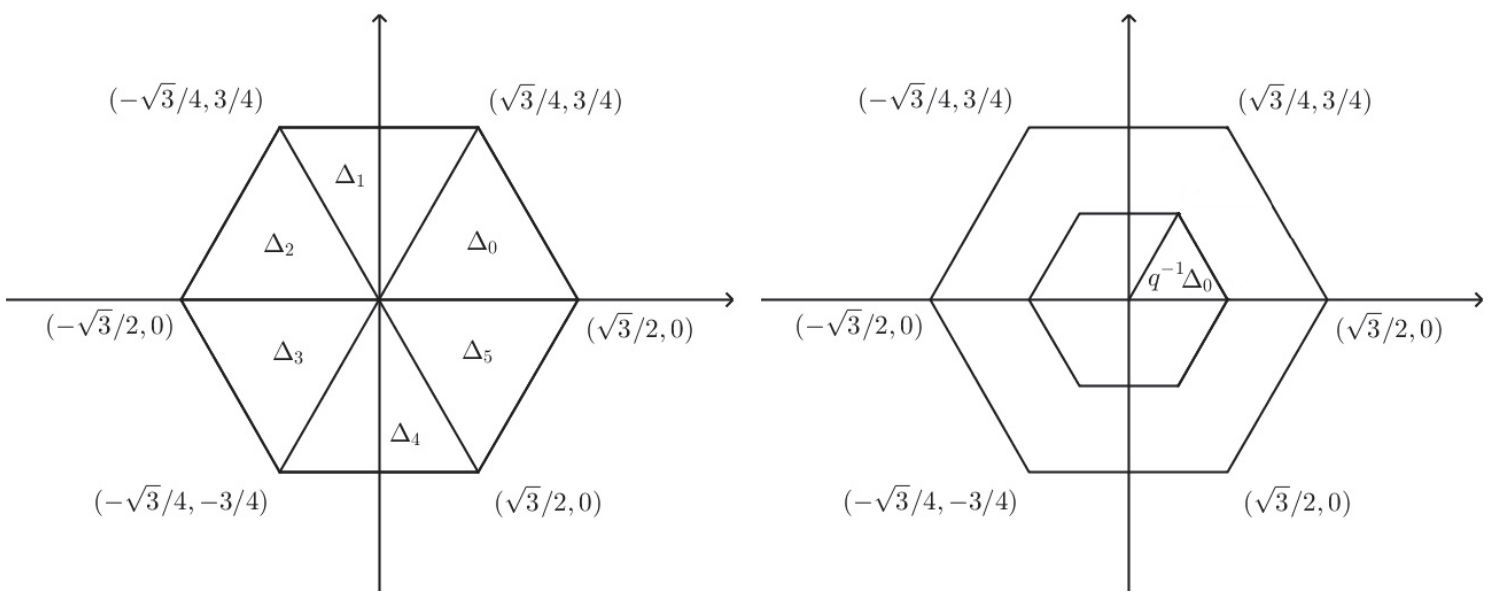

Figure 2

and $b_{i}=-b_{i-4}$ for $4 \leq i \leq 7$. Let $\Delta_{0}$ be the triangular region with vertices in $(0,0),(1 / 2,0)$, and $(1 / 2,1 / 2)$; we denote $\Delta_{i}=b_{i} \Delta_{0}$ for $1 \leq i \leq 7$. If $\varphi=2 \sqrt{2} \chi_{\Delta_{0}}$, then the system

$$
\left\{D_{b} T_{k} \varphi: b \in B, k \in \mathbb{Z}^{2}\right\}
$$

is an orthogonal basis for its closed span $V_{0}$. The space $V_{0}$ is the subspace of $\mathscr{L}^{2}\left(\mathbb{R}^{2}\right)$, consisting of all square integrable functions that are constant on each $\mathbb{Z}^{2}$ translate of the triangles $\Delta_{i}, 1 \leq i \leq 7$. Thus, the spaces $V_{j}=D_{q^{-j}} V_{0}, j \in \mathbb{Z}$, consist of all functions in $\mathscr{L}^{2}\left(\mathbb{R}^{2}\right)$, which are constant in each $q^{-j} \mathbb{Z}^{2}$-translate of the triangles $q^{-1} \Delta_{i}, 1 \leq i \leq 7$, in consequence $V_{j} \subset V_{j+1}$. Hence, $\left\{V_{j}\right\}$ is an $A B$-MRA with $\varphi$ as a scaling function.

Figure 1 is reproduced from Krishtal et al. (2007) [under the Creative Commons Attribution License/public domain].

(2) Let $B$ be the group generated by the matrix $\rho=$ $(1 / 2)\left(\begin{array}{cc}1 & -\sqrt{3} \\ \sqrt{3} & 1\end{array}\right)$. This group has order of 6 and is the counter-clockwise rotation by $\pi / 3$ radians. Consider the hexagon with vertices in set

$$
\left\{\left( \pm \frac{\sqrt{3}}{2}, 0\right),\left(\frac{\sqrt{3}}{4}, \pm \frac{3}{4}\right),\left(-\frac{\sqrt{3}}{4}, \pm \frac{3}{4}\right)\right\} .
$$

Let $\Delta_{0}$ be the triangle with vertices in $(0,0),(\sqrt{3} / 2,0)$, and $(\sqrt{3} / 4,3 / 4)$. We define $\Delta_{i}=\rho^{i} \Delta_{0}$ for $0 \leq i \leq 5$. We take $c=(1 / 4)\left(\begin{array}{cc}0 & 3 \sqrt{3} \\ 6 & 3\end{array}\right)$ and we define $\Gamma=c \mathbb{Z}^{2}$. The set of all functions that are constant on $\Gamma$-translates of triangles $\Delta_{i}, 0 \leq i \leq 5$, is the space $V_{0} \subset \mathscr{L}^{2}\left(\mathbb{R}^{2}\right)$. Moreover, the elements of $\Gamma$ translate the center of the hexagon to the point of $\Gamma$, and so we have a partition of $\mathbb{R}^{2}$. Let $q=\left(\begin{array}{cc}1 & -\sqrt{3} \\ \sqrt{3} & 1\end{array}\right)$ and the function $\varphi=E \chi_{\Delta_{0}}$, with $E=4 / \sqrt[4]{27}$. The MRA $\left\{V_{j}\right\}$ is obtained by the system

$$
\left\{D_{q^{j}} D_{\rho^{i}} T_{\gamma} \varphi: j \in \mathbb{Z}, 0 \leq i \leq 5, \gamma \in \Gamma\right\}
$$

where the spaces $V_{j}$ are $q^{-j}$-dilatation of $V_{0}$, for $j \in \mathbb{Z}$. Figure 2 can be found in [6]. 


\section{Approximation of Measures Using Multiresolution Analysis}

3.1. Absolutely Continuous Measures with respect to Lebesgue Measures. We consider the following conditions:

(A1) $X$ is a compact subset of $\mathbb{R}^{n}$.

(A2) The measure $\mu$ is absolutely continuous with respect to $\lambda$, where $\lambda$ is the Lebesgue measure.

Condition (A2) guarantees the existence of functions $f \in$ $\mathscr{L}^{1}(X)$, where $f$ is the Radon-Nikodym derivative with respect to Lebesgue measure $\lambda$.

In this analysis, we also assume the following extra condition:

(A3) The functions $f$ also belong in $\mathscr{L}^{2}\left(X_{k}\right)$.

Notice that

$$
f \in \mathscr{L}^{1}(X) \cap \mathscr{L}^{2}(X) \subset \mathscr{L}^{2}\left(\mathbb{R}^{n}\right) .
$$

Let $\left\{V_{j}\right\}$ be an $A B$-MRA on $\mathbb{R}^{n}$; the elements of $\left\{V_{j}\right\}$ are given by scaling functions $\varphi=E_{1} \chi_{\Delta}$, the latice $\Gamma$, finite group $B_{1}$, and dilatation $A=\left\{a_{1}^{j}\right\}$

Remark 2. Note that the classical multiresolution analysis of Haar on $\mathscr{L}^{2}\left(\mathbb{R}^{2}\right)$ is a particular case of $A B$-MRA on $\mathbb{R}^{2}$, where the complete lattice is $\Gamma=\mathbb{Z}^{2}$, the group $B$ is trivial, and the dilation associated is $A=\left\{2^{-j}(1,1)\right\}$. In this case, we have that scaling function is $\varphi_{\Delta}$, where $\Delta=[0,1] \times[0,1]$ is the fundamental domain associated with the action of $\Gamma$ on $\mathbb{R}^{2}$.

We denote by $P_{j}$ the projection from $\mathscr{L}^{2}\left(\mathbb{R}^{n}\right)$ into $V_{j}$, which is given by

$$
P_{j} f=\sum_{\alpha \in(B \Gamma)^{j}}\left\langle f, \varphi_{\alpha}^{j}\right\rangle \varphi_{\alpha}^{j}
$$

for all $f \in \mathscr{L}^{2}\left(\mathbb{R}^{n}\right)$. Moreover, if the function $f$ has support in $X$, then the above sum is finite, since $X$ is compact.

From now on, we shall only functions with support in the compact set $X$ and we have $\mathscr{L}^{2}(X) \subset \mathscr{L}^{2}\left(\mathbb{R}^{n}\right)$; that is, each function $f \in \mathscr{L}^{2}(X)$ can be considered in $\mathscr{L}^{2}\left(\mathbb{R}^{n}\right)$, where $f(x)=0$ if $x \notin X$.

We know that the $A B$-MRA is dense in $\mathscr{L}^{2}(X)$; thus we have that

$$
\left\|P^{j} f-f\right\|_{2} \longrightarrow 0, \quad \text { when } j \longrightarrow \infty
$$

Using the fact that $X$ is compact, we obtain that

$$
\left\|P_{j} f-f\right\|_{1} \leq \lambda(X)^{1 / 2}\|P j f-f\|_{2}
$$

From the above equation, we define the approximation of the measure to the level $j$ by

$$
\mathrm{d} \mu_{j}=P_{j} f \mathrm{~d} \lambda,
$$

which are measures in $X$ for each $j$. Now we want to prove that these measures are probability measures.
Definition 3. The expectation $\mathbb{E}$ with respect to Lebesgue measure $\lambda$ of function $f$ is defined by

$$
\mathbb{E}[f]=\int_{\mathbb{R}^{n}} f \mathrm{~d} \lambda,
$$

where $f$ is a Lebesgue measurable function. Also, the conditional expectation of $f$ given $A$, with $A$ being a Lebesgue measurable set, is defined by $\mathbb{E}[f \mid A]=\mathbb{E}\left[f \cdot \chi_{A}\right]$.

In particular, the expectation on the Lebesgue measure $\lambda$ satisfies the following property.

Theorem 4. Consider that $j \in \mathbb{Z}$ is fixed and $f \in \mathscr{L}^{2}\left(\mathbb{R}^{n}\right)$. Then $\mathbb{E}\left[P_{j} f\right]=\mathbb{E}[f]$, where $P_{j}$ is the projection of the level $j$ associated with $A B-M R A\left\{V_{j}\right\}$.

Proof. We take $f \in \mathscr{L}^{2}\left(\mathbb{R}^{n}\right)$; now we calculate $\mathbb{E}\left[P_{j} f\right]$; thus

$$
\begin{aligned}
\mathbb{E}\left[P_{j} f\right] & =\int_{\mathbb{R}^{n}} \sum_{\alpha \in B \Gamma}\left\langle f, \varphi_{\alpha}^{j}\right\rangle \varphi_{\alpha}^{j} \mathrm{~d} \lambda \\
& =\sum_{\alpha \in B \Gamma}\left\langle f, \varphi_{\alpha}^{j}\right\rangle \int_{\mathbb{R}^{n}} \varphi_{\alpha}^{j} \mathrm{~d} \lambda
\end{aligned}
$$

We have that the functions $\varphi_{\alpha}^{j}=E_{j} \chi_{\Delta_{\alpha}^{j}}$, where $\Delta_{\alpha}^{j}$ is the translation and dilation of the fundamental region $\Delta$ and $E_{j}=\lambda\left(\Delta_{\alpha}^{j}\right)^{-1 / 2}$; this value does not depend on $\alpha$. From this, we have that

$$
\int_{\mathbb{R}^{n}} \varphi_{k}=\int_{\Delta_{k}} \varphi_{k}=E_{j}^{-1} .
$$

Using the above equations, we have that

$$
\mathbb{E}\left[P_{j} f\right]=\sum_{\alpha \in B \Gamma}\left\langle f, \chi_{\Delta_{\alpha}^{j}}\right\rangle .
$$

Moreover, using the fact that $\Delta_{\alpha}^{j} \cap \Delta_{\beta}^{j}=\emptyset$ for $\alpha \neq \beta$, it is clear that

$$
\mathbb{E}\left[P_{j} f\right]=\left\langle f, \sum_{\alpha \in B \Gamma} \chi_{\Delta_{\alpha}^{j}}\right\rangle=\left\langle f, \chi_{\mathbb{R}^{n}}\right\rangle=\mathbb{E}[f] .
$$

As immediate consequence of the previous theorem, we get the following result.

Corollary 5. We suppose that the measure $\mu$ on $X$ satisfies (A1)-(A2); then the sequences of probability measures $\left\{\mu^{j}\right\} \subset$ $M^{+}(X)$ given by (15) converge to $\mu$ in $\mathscr{L}^{1}$ and $\mathscr{L}^{2}$ sense.

3.2. Non-Absolutely-Continuous Measures with respect to Lebesgue Measure. Now, we consider that $\mu$ is a probability measure on the compact set $X$, which is unnecessarily absolutely continuous measure with respect to Lebesgue measure $\lambda$. Note that each element of sequence (15) can be written by

$$
\mu^{j}(U)=\sum_{\alpha \in B \Gamma} \mu\left(\Delta_{\alpha}^{j}\right) \frac{\lambda\left(U \cap \Delta_{\alpha}^{j}\right)}{\lambda\left(\Delta_{\alpha}^{j}\right)}
$$


for all Borel measurable sets $U$ on $X$. Moreover, these approximations are well defined for every measure $\mu$ on $X$.

Definition 6. Let $\left\{\mu_{n} ; n \geq 0\right\}$ be a sequence of probability measures on a metric space $(X, d)$. We say that $\mu_{n}$ converges weakly to $\mu$ and denote $\mu_{n} \rightarrow{ }_{w} \mu$ if $\mu_{n}(f) \rightarrow \mu(f)$ as $n \rightarrow \infty$ for all bounded continuous functions $f$ on $M$, where $\mu(f)=$ $\int_{M} f d \mu$.

Theorem 7. Given a measure $\mu$ on the compact set $X$, the sequence of measures $\left\{\mu^{j}\right\}$ on $X$ defined in (21) converge weakly to measure $\mu$.

Proof. Let $f: X \rightarrow \mathbb{R}$ be a continuous and bounded real function. From the fact that $X$ is compact, we obtain that $f$ is absolutely continuous function on $X$; thus, given $\epsilon>0$, there exists $\delta>0$ such that

$$
|x-y|<\delta \text { implies }|f(x)-f(y)|<\frac{\epsilon}{2}
$$

We take $j_{0} \in \mathbb{Z}$ such that for all $x, y \in \Delta_{\alpha}^{j}$ implies $|x-y|<\delta$ for all $j \geq j_{0}$ and $\alpha \in B \Gamma$. Moreover, we know that there exist $\alpha_{1}, \ldots, \alpha_{r} \in B \Gamma$ such that

$$
X \subset \bigcup_{m=1}^{r} \Delta_{\alpha_{m}}^{j} .
$$

In consequence, we obtain the following relations:

$$
\begin{aligned}
& \left|\int_{X} f \mathrm{~d} \mu-\int_{X} f \mathrm{~d} \mu^{j}\right| \leq\left|\int_{\cup \Delta_{\alpha_{m}}^{j}} f \mathrm{~d} \mu-\int_{\cup \Delta_{\alpha_{m}}^{j}} f \mathrm{~d} \mu^{j}\right| \\
& \quad \leq \sum_{m=1}^{r}\left|\int_{\Delta_{\alpha_{m}}^{j}} f \mathrm{~d} \mu-\int_{\Delta_{\alpha_{m}}^{j}} f \mathrm{~d} \mu^{j}\right| \\
& \quad=\sum_{m=1}^{r}\left|\int_{\Delta_{\alpha_{m}}^{j}} f \mathrm{~d} \mu-\int_{\Delta_{\alpha_{m}}^{j}} f \frac{\mu\left(\Delta_{\alpha_{m}}^{j}\right)}{\lambda\left(\Delta_{\alpha_{m}}^{j}\right)} \mathrm{d} \lambda\right| ;
\end{aligned}
$$

we take an element $x_{\alpha_{m}}^{j} \in \Delta_{\alpha_{m}}^{j}$ to obtain the following relations:

$$
\begin{gathered}
\mid \int_{\Delta_{\alpha_{m}}^{j}} f \mathrm{~d} \mu-\int_{\Delta_{\alpha_{m}}^{j}} f\left(x_{\alpha_{m}}^{j}\right) \mathrm{d} \mu+f\left(x_{\alpha_{m}}^{j}\right) \mu\left(\Delta_{\alpha_{m}}^{j}\right) \\
-\int_{\Delta_{\alpha_{m}}^{j}} f \frac{\mu\left(\Delta_{\alpha_{m}}^{j}\right)}{\lambda\left(\Delta_{\alpha_{m}}^{j}\right)} \mathrm{d} \lambda\left|\leq \int_{\Delta_{\alpha_{m}}^{j}}\right| f-f\left(x_{\alpha_{m}}^{j}\right) \mid \mathrm{d} \mu \\
+\frac{\mu\left(\Delta_{\alpha_{m}}^{j}\right)}{\lambda\left(\Delta_{\alpha_{m}}^{j}\right)} \int_{\Delta_{\alpha_{m}}^{j}}\left|f-f\left(x_{\alpha_{m}}^{j}\right)\right| \mathrm{d} \lambda
\end{gathered}
$$

Therefore,

$$
\begin{aligned}
& \left|\int_{X} f \mathrm{~d} \mu-\int_{X} f \mathrm{~d} \mu^{j}\right| \leq \sum_{m=1}^{r}\left[\int_{\Delta_{\alpha_{m}}^{j}}\left|f-f\left(x_{\alpha_{m}}^{j}\right)\right| \mathrm{d} \mu\right. \\
& \left.+\frac{\mu\left(\Delta_{\alpha_{m}}^{j}\right)}{\lambda\left(\Delta_{\alpha_{m}}^{j}\right)} \int_{\Delta_{\alpha_{m}}^{j}}\left|f-f\left(x_{\alpha_{m}}^{j}\right)\right| \mathrm{d} \lambda\right]
\end{aligned}
$$

$$
\begin{aligned}
& <\sum_{m=1}^{r}\left[\frac{\epsilon}{2} \int_{\Delta_{\alpha_{m}}^{j}} \mathrm{~d} \mu+\frac{\mu\left(\Delta_{\alpha_{m}}^{j}\right)}{\lambda\left(\Delta_{\alpha_{m}}^{j}\right)} \frac{\epsilon}{2} \int_{\Delta_{\alpha_{m}}^{j}} \mathrm{~d} \lambda\right]=\frac{\epsilon}{2} \\
& \cdot \sum_{m=1}^{r}\left[\mu\left(\Delta_{\alpha_{m}}^{j}\right)+\frac{\mu\left(\Delta_{\alpha_{m}}^{j}\right)}{\lambda\left(\Delta_{\alpha_{m}}^{j}\right)} \lambda\left(\Delta_{\alpha_{m}}^{j}\right)\right] \\
& =\epsilon \sum_{m=1}^{r} \mu\left(\Delta_{\alpha_{m}}^{j}\right)=\epsilon \cdot \mu(X)=\epsilon .
\end{aligned}
$$

\section{Discretization of the Monge-Kantorovich Problem Using Multiresolution Analysis}

Let $M(X \times Y)$ be the linear space of finite signed on $X \times Y$ and let $M^{+}(X \times Y)$ be the set of all nonnegative measures in $M(X \times Y)$. Given $\mu \in M(X \times Y)$, we denote by $\Pi_{1} \mu$ and $\Pi_{2} \mu$ the marginal of $\mu$ on $X$ and $Y$, respectively; that is,

$$
\begin{aligned}
& \Pi_{1} \mu(A \times Y)=\nu_{1}(A), \\
& \Pi_{2} \mu(X \times B)=v_{2}(B)
\end{aligned}
$$

for all sets $A$ and $B$, such that $\nu_{1}$ and $\nu_{2}$ are measurable, respectively.

The Monge-Kantorovich mass transfer problem is given as follows:

$$
\begin{aligned}
\text { MK: minimize } & \langle\mu, c\rangle:=\int c \mathrm{~d} \mu \\
\text { subject to: } & \Pi_{1} \mu=v_{1}, \\
& \Pi_{2} \mu=v_{2}, \\
& \mu \in M^{+}(X \times Y)
\end{aligned}
$$

A measure $\mu \in M(X \times Y)$ is said to be a feasible solution for MK problem if it satisfies (28) and $\langle\mu, c\rangle$ is finite. The MK problem is called consistent if the set of feasible solutions is nonempty, in which case its optimal value is defined as

$$
\begin{aligned}
& \inf (\mathrm{MK}) \\
& \quad=\inf \{\langle\mu, c\rangle: \mu \text { is a feasible solution for } \mathrm{MK}\} .
\end{aligned}
$$

It is said that the MK problem is solvable if there is a feasible solution $\mu^{*}$ that attains the optimal value. In this case, $\mu^{*}$ is called an optimal solution for the MK problem and the value $\inf (\mathrm{MK})$ is written as $\min (\mathrm{MK})=\left\langle\mu^{*}, c\right\rangle$.

Note that since $\nu_{1}$ and $\nu_{2}$ are probability measures, a feasible solution for $\mathrm{MK}$ is also a probability measure. Moreover, if $c$ is a continuous function on $X \times Y$ and $X$ and $Y$ are compact subsets on $\mathbb{R}^{n}$, then the product measure $\mu:=\nu_{1} \times \nu_{2}$ is a feasible solution. Therefore the MK problem is consistent, and so the $\mathrm{MK}$ problem is solvable in this case.

We assume the following conditions: $X$ and $Y$ are compact subsets of $\mathbb{R}^{n}$ and $c: X \times Y \rightarrow \mathbb{R}$ is a bounded continuous function. 
Let $\left\{V_{j}\right\}$ and $\left\{V_{j}^{\prime}\right\}$ be $A B$-MRA on $\mathbb{R}^{n}$ with scaling functions $\varphi_{1}=E_{1} \chi_{\Delta_{1}}$ and $\varphi_{2}=E_{2} \chi_{\Delta_{2}}$, lattices $\Gamma_{1}$ and $\Gamma_{2}$, finite groups $B_{1}$ and $B_{2}$, and dilatations $A_{1}=\left\{a_{1}^{j}\right\}$ and $A_{2}=\left\{a_{2}^{j}\right\}$, respectively. We can obtain a new $A B$-MRA $\left\{V_{j}^{*}\right\}$ on $\mathbb{R}^{2 n}$ with scaling functions $\varphi=\varphi_{1} \varphi_{2}=E_{1} E_{2} \chi_{\Delta_{1} \times \Delta_{2}}$, lattice $\Gamma=\Gamma_{1} \times \Gamma_{2}$, finite group $B=B_{1} \times B_{2}$, and dilatation $\left\{a_{1}^{j} \times a_{2}^{j}\right\}$. The projections of these $A B$-MRA to the level $j$ are denoted by $P_{1}^{j}, P_{2}^{j}$, and $Q^{j}$.

Now we proceed to discretization of the MK-problem using the results of Section 2; then we have that $\mu^{j}, v_{1}^{j}$, and $v_{2}^{j}$ are the projections to level $j$ of the respective $A B$-MRA; thus, using these measures, we obtain the discretization of the MK-problem in the level $j$, which is given by

$$
\begin{aligned}
\text { MK }^{j} \text { minimize } & \left\langle\mu^{j}, c\right\rangle:=\int c \mathrm{~d} \mu^{j} \\
\text { subject to: } & \Pi_{1} \mu^{j}=v_{1}^{j}, \\
& \Pi_{2} \mu^{j}=v_{2}^{j}, \\
& \mu^{j} \in M^{+}(X \times Y) .
\end{aligned}
$$

We shall give explicit expressions for the discretization of the measures and the cost function associated with MKproblem using the $A B-\mathrm{MRA}$, which are given by

$$
\begin{aligned}
& \mu^{j}=\sum_{(\alpha, \beta) \in(B \Gamma)_{0}^{j}} \mu_{\alpha, \beta}^{j} \varphi_{1, \alpha}^{j}(x) \varphi_{2, \beta}^{j}(y), \\
& \quad \text { with } \mu_{\alpha, \beta}^{j}=\left\langle\mu, \varphi_{1, \alpha}^{j} \varphi_{2, \beta}^{j}\right\rangle \geq 0 \\
& c^{j}=\sum_{(\alpha, \beta) \in(B \Gamma)_{0}^{j}} c_{\alpha, \beta}^{j} \varphi_{1, \alpha}^{j}(x) \varphi_{2, \beta}^{j}(y), \\
& \quad \text { with } c_{\alpha, \beta}^{j}=\left\langle c, \varphi_{1, \alpha}^{j} \varphi_{2, \beta}^{j}\right\rangle \\
& v_{1}^{j}=\sum_{\alpha \in\left(B \Gamma_{1}\right)_{0}^{j}} a_{\alpha}^{j} \varphi_{1, \alpha}^{j}(x), \quad \text { with } a_{\alpha}^{j}=\left\langle v_{1}, \varphi_{1, \alpha}^{j}\right\rangle \\
& v_{2}^{j}=\sum_{\beta \in\left(B \Gamma_{2}\right)_{0}^{j}} b_{\beta}^{j} \varphi_{2, \beta}^{j}(y), \quad \text { with } b_{\beta}^{j}=\left\langle v_{2}, \varphi_{2, \beta}^{j}\right\rangle
\end{aligned}
$$

where $\mu_{\alpha, \beta}^{j}, a_{\alpha, \beta}^{j}$, and $b_{\alpha, \beta}$ are nonnegative real numbers. Now we calculate $\left\langle\mu^{j}, c\right\rangle$; thus

$$
\left\langle\mu^{j}, c\right\rangle=\left\langle\mu^{j}, Q^{j} c+\left(Q^{j} c\right)^{\perp}\right\rangle=\left\langle Q^{j} c, \mu^{j}\right\rangle,
$$

and hence, using the above equation and the orthonormality of family $\left\{\varphi_{1, \alpha}^{j} \varphi_{2, \beta}^{j}\right\}$, we obtain

$$
\left\langle\mu^{j}, c\right\rangle=\sum_{(\alpha, \beta) \in(B \Gamma)_{0}^{j}} c_{\alpha, \beta}^{j} \cdot \mu_{(\alpha, \beta)}^{j} .
$$

We consider that $\alpha_{i}$ is a fixed element in $\left(B \Gamma_{1}\right)_{0}^{j}$ and we have that $\Delta_{1, \alpha_{i}}^{j}$ is the region associated with $\alpha_{i}$ for $i=1, \ldots, r$; then we obtain

$$
\begin{aligned}
\mu^{j}\left(\Delta_{1, \alpha_{i}}^{j} \times \mathbb{R}^{n}\right)=\int_{\Delta_{1, \alpha_{i}}^{j} \times \mathbb{R}^{n}} \mathrm{~d} \mu^{j} \\
=\int_{\mathbb{R}^{2 n}} \chi_{\Delta_{1, \alpha_{i}}^{j}} \\
\quad \cdot \chi_{\mathbb{R}^{n}} \sum_{(\alpha, \beta) \in B \Gamma} \mu_{\alpha, \beta}^{j} \varphi_{1, \alpha}^{j}(x) \varphi_{2, \beta}^{j}(y) \mathrm{d} \lambda(x) \mathrm{d} \lambda(y) \\
=\sum_{(\alpha, \beta) \in B \Gamma} \mu_{\alpha, \beta}^{j} \int_{\mathbb{R}^{n}}\left(\chi_{\Delta_{1, \alpha_{i}}^{j}} \cdot E_{1} \chi_{\Delta_{1, \alpha}^{j}}\right)(x) \mathrm{d} \lambda(x) \\
\quad \cdot \int_{\mathbb{R}^{n}}\left(\chi_{\mathbb{R}^{n}} \cdot E_{2} \chi_{\Delta_{2, \beta}^{j}}\right)(y) \mathrm{d} \lambda(y)=\sum_{\beta \in\left(B \Gamma_{2}\right)_{0}^{j}} \mu_{\alpha_{i}, \beta}^{j} .
\end{aligned}
$$

On the other hand, we have that

$$
\begin{aligned}
\nu_{1}^{j}\left(\Delta_{1, \alpha_{i}}^{j}\right) & =\int_{\Delta_{1, \alpha_{i}}^{j}} P_{1}^{j} f_{1}(x) \mathrm{d} \lambda(x) \\
& =\int_{\Delta_{1, \alpha_{i}}^{j}} \sum_{\alpha \in\left(B \Gamma_{1}\right)_{0}^{j}} a_{\alpha}^{j} \varphi_{1, \alpha}^{j}(x) \\
& =\sum_{\alpha \in\left(B \Gamma_{1}\right)_{0}^{j}} a_{\alpha}^{j} \int_{\mathbb{R}^{n}}\left(\chi_{\Delta_{1, \alpha_{i}}^{j}} \cdot E_{1} \chi_{\Delta_{1, \alpha}^{j}}\right)(x) \mathrm{d} \lambda(x) \\
& =a_{\alpha_{i}}^{j}
\end{aligned}
$$

From the above equations, we have that the condition $\mu^{j}\left(\Delta_{1, \alpha_{i}}^{j} \times \mathbb{R}^{n}\right)=v_{1}^{j}\left(\Delta_{1, \alpha_{i}}^{j}\right)$ is equivalent to

$$
\sum_{\beta \in\left(B \Gamma_{2}\right)_{0}^{j}} \mu_{\alpha_{i}, \beta}^{j}=a_{\alpha_{i}}^{j}, \quad i=1, \ldots, r .
$$

Analogously, for all the elements $\beta_{1}, \ldots, \beta_{s}$ of $\left(B \Gamma_{2}\right)_{0}^{j}$, we have that

$$
\sum_{\alpha \in\left(B \Gamma_{1}\right)_{0}^{j}} \mu_{\alpha, \beta_{k}}^{j}=b_{\beta_{k}}^{j}, \quad k=1, \ldots, s .
$$

Using Theorem 4, we obtain that

$$
\sum_{(\alpha, \beta) \in(B \Gamma)_{0}^{j}} \mu_{\alpha, \beta}^{j}=\sum_{k=1}^{r} a_{\alpha_{k}}^{j}=\sum_{k=1}^{s} b_{\beta_{k}}^{j}=1
$$

In summary, we have that the $M K^{j}$ problem given by (30) is equivalent to the following problem:

$$
\begin{aligned}
\text { MKD }^{j} \text { : minimize } & \sum_{(\alpha, \beta) \in(B \Gamma)_{0}^{j}} \mu_{\alpha, \beta}^{j} \cdot c_{\alpha, \beta}^{j} \\
\text { subject to: } & \sum_{\beta \in\left(B \Gamma_{2}\right)_{0}^{j}} \mu_{\alpha_{k}, \beta}^{j}=a_{\alpha_{k}}^{j} \quad k=1, \ldots r \\
& \sum_{\alpha \in\left(B \Gamma_{1}\right)_{0}^{j}} \mu_{\alpha, \beta_{k}}^{j}=b_{\beta_{k}}^{j}, \quad k=1, \ldots s
\end{aligned}
$$




$$
\begin{gathered}
\sum_{(\alpha, \beta) \in(B \Gamma)_{0}^{j}} \mu_{\alpha, \beta}^{j}=1 \\
\mu_{\alpha, \beta}^{j} \geq 0, \quad \forall(\alpha, \beta) \in(B \Gamma)_{0}^{j}
\end{gathered}
$$

The problem $\mathrm{MKD}^{j}$, given the above system, has a feasible solution, so we know that $c$ is bounded. Then we have that the problem $\mathrm{MKD}^{j}$ has an optimal solution; for more details, see Chapter 10 in [7].

Let $\mu_{*}$ be the optimal solution of MK problem given in (28). We use the optimal solution $\mu_{*}$ to induce a sequence of measures $\mu_{*}^{j}$ such that $\mu_{*}^{j}=Q^{j} \mu_{*}$ (see Section 3.2). Let $\eta_{*}^{j}$ be the optimal solution of $\mathrm{MK}^{j}$ problem given in (30).

For $j \in \mathbb{Z}$, we defined the following $\sigma$-algebras:

$$
\begin{array}{ll}
\mathscr{F}_{1}^{j}=\left\langle\Delta_{1, \alpha}^{j}\right\rangle, & \forall \alpha \in B \Gamma_{1}^{j} \\
\mathscr{F}_{2}^{j}=\left\langle\Delta_{2, \beta}^{j}\right\rangle, & \forall \beta \in B \Gamma_{2}^{j} \\
\mathscr{F}^{j}=\left\langle\Delta_{\alpha, \beta}^{j}\right\rangle, & \forall(\alpha, \beta) \in B \Gamma^{j}
\end{array}
$$

where $\left\langle A_{i}\right\rangle_{i \in I}$ denotes the $\sigma$-algebra generated by the sets $A_{i}$ with $i \in I$ and the lattices $B \Gamma_{1}^{j}, B \Gamma_{2}^{j}$, and $B \Gamma^{j}$ are defined as in (3).

Definition 8. Let $\mathscr{F}^{\prime}$ be a sub- $\sigma$-algebra of $\mathscr{F}$, and let $X \in \mathscr{L}^{1}$ be a random variable. We say that the random variable $X^{\prime}$ is the conditional expectation of $X$ with respect to $\mathscr{F}^{\prime}$ and denote it by $\mathbb{E}\left[X \mid \mathscr{F}^{\prime}\right]$ if and only if

(i) $X^{\prime} \in \mathscr{L}^{1}$.

(ii) $X^{\prime}$ is $\mathscr{F}^{\prime}$-measurable.

(iii) $\mathbb{E}\left[X^{\prime} \chi_{A}\right]=\mathbb{E}\left[X \chi_{A}\right]$, for all $a \in \mathscr{F}^{\prime}$.

Remark 9. Given $f \in \mathscr{L}^{1}\left(X_{1} \times X_{2}\right)$, we have that

$$
\mathbb{E}\left[f \mid \mathscr{F}^{j}\right]=\mathbb{E}\left[Q^{j}(f)\right] .
$$

There are analogous results for $P_{1}^{j}$ and $P_{2}^{j}$ through the conditional expectations of $\mathscr{F}_{1}^{j}$ and $\mathscr{F}_{2}^{j}$, respectively.

Proposition 10. Let $\mu$ be a feasible solution of the $M K$ problem; then $\mu^{j}$ is a feasible solution of the $M K D^{j}$-problem.

Proof. We suppose that $\mu$ satisfies (28). Then we have the following relations:

$$
\begin{aligned}
\Pi_{1} \mu^{j}(E)=\mu_{*}^{j}\left(E \times \mathbb{R}^{n}\right)=\int_{E \times \mathbb{R}^{n}} \mathrm{~d} \mu_{*}^{j} \\
=\int_{E} \int_{\mathbb{R}^{n}} \sum_{(\alpha, \beta) \in(B \Gamma)_{0}^{j}} \mu_{\alpha, \beta}^{j} \varphi_{1, \alpha}^{j}(x) \varphi_{2, \beta}^{j}(y) \mathrm{d}(x) \mathrm{d}(y) \\
=\int_{E} \sum_{(\alpha, \beta) \in(B \Gamma)_{0}^{j}} \mu_{\alpha, \beta}^{j} \varphi_{1, \alpha}^{j}(x) \int_{\mathbb{R}^{n}} \varphi_{2, \beta}^{j}(y) \mathrm{d}(y) \mathrm{d}(x) \\
=\int_{E} \sum_{(\alpha, \beta) \in(B \Gamma)_{0}^{j}} \mu_{\alpha, \beta}^{j} \varphi_{1, \alpha}^{j}(x)\left(E_{2}^{j}\right)^{-1} \mathrm{~d}(x)
\end{aligned}
$$

$$
\begin{aligned}
& =\int_{E} \sum_{(\alpha, \beta) \in(B \Gamma)_{0}^{j}} \int_{\Delta_{1, \alpha} \times \Delta_{2, \beta}} E_{1}^{j} \mathrm{~d} \mu \varphi_{1, \alpha}^{j}(x) \mathrm{d}(x) \\
& =\int_{E} \sum_{\alpha \in \Gamma_{1}} \sum_{\beta \in\left[(B \Gamma)_{0}^{j}\right]_{\alpha}}\left(E_{1}^{j}\right)^{2} \chi_{\Delta_{1, \alpha}^{j}}(x) \mathrm{d}(x) \\
& =\int_{E} \sum_{\alpha \in \Gamma_{1}}\left[\int_{\Delta_{1, \alpha} \times \mathbb{R}^{n}} \mathrm{~d} \mu\right]\left(E_{1}^{j}\right)^{2} \chi_{\Delta_{1, \alpha}^{j}}(x) \mathrm{d}(x) \\
& =\nu_{1}^{j}(E)
\end{aligned}
$$

where $\left[(B \Gamma)_{0}^{j}\right]_{\alpha}=\left\{(\alpha, \beta) \in(B \Gamma)_{0}^{j}: \alpha\right.$ is fixed $\}$ and $E$ a measurable set. Similarly, we obtain that $\Pi_{2} \mu^{j}(E)=v_{2}^{j}(E)$. Therefore the result is as follows.

Remark 11. If $\mu_{*}$ is an optimal solution of the MK problem, then $\mu_{*}^{j}$ is a feasible solution of the $\mathrm{MKD}^{j}$-problem.

Proposition 12. Given $E \in \mathscr{F}_{1}^{j}$ and $F \in \mathscr{F}_{2}^{j}$, one has

$$
\begin{aligned}
\eta^{j}\left(E \times \mathbb{R}^{n}\right) & =\mu\left(E \times \mathbb{R}^{n}\right), \\
v_{1}^{j}(E) & =\nu_{1}(E) \\
\eta^{j}\left(\mathbb{R}^{n} \times F\right) & =\mu\left(\mathbb{R}^{n} \times F\right), \\
v_{2}^{j}(F) & =\nu_{2}(F),
\end{aligned}
$$

where $\mu$ and $\eta^{j}$ are feasible solutions of the $M K$ and $M K^{j}$ problems, respectively.

Proof. Let $\Delta_{\alpha}^{j}$ be a generator element of $\sigma$-algebra $\mathscr{F}^{j}$. Then

$$
\begin{aligned}
\mu\left(\Delta_{\alpha}^{j} \times \mathbb{R}^{j}\right) & =\Pi_{1} \mu\left(\Delta_{\alpha}^{j}\right)=\nu_{1}\left(\Delta_{\alpha}^{j}\right)=v_{1}^{j}\left(\Delta_{\alpha}^{j}\right) \\
& =\Pi_{1} \eta^{j}\left(\Delta_{\alpha}^{j}\right)=\eta^{j}\left(\Delta_{\alpha}^{j} \times \mathbb{R}^{j}\right)
\end{aligned}
$$

In above equation, we use the fact that $\nu_{1}^{j}(E)=\nu_{1}(E)$ for all $E \in \mathscr{F}^{j}$ in consequence $\eta^{j}\left(E \times \mathbb{R}^{n}\right)=\mu\left(E \times \mathbb{R}^{n}\right)$ for all $E \in \mathscr{F}_{1}^{j}$. Analogously, we have that $\eta^{j}\left(\mathbb{R}^{n} \times F\right)=\mu\left(\mathbb{R}^{n} \times F\right)$.

Definition 13. Let $(X, \tau)$ be a topological space, and let $\mathscr{F}$ be $\sigma$-algebra on $X$ that contains the topology $\tau$. Let $\mathscr{M}$ be a collection of measures defined on $\mathscr{F}$. The collection $\mathscr{M}$ is called tight if, for any $\epsilon>0$, there is a compact subset $K_{\epsilon}$ of $X$ such that, for all measures $\mu \in \mathscr{M},|\mu|\left(X \backslash K_{\epsilon}\right)<\epsilon$, where $|\mu|$ is the total variation measure of $\mu$.

Proposition 14. Consider the sequence $\left\{\eta_{*}^{j}\right\}$ of probability measures, where $\eta_{*}^{j}$ is the optimal solution of $M K^{j}$ for each $j \in \mathbb{Z}$. Then there exists a subsequent $\left\{\eta_{*}^{j_{n}}\right\}$ and a probability measure $\eta_{*}$ such that $\mu_{*}^{j_{n}} \rightarrow \mu_{*}$ weakly. Moreover, the probability measure $\eta_{*}$ is an optimal solution of $M K$.

Proof. We know that $X_{1}$ and $X_{2}$ are compact sets, provided that the measures $\left\{\mu_{*}^{j}\right\}$ are tight for each $j \in \mathbb{Z}$. Now, using 
Prokhorov's Theorem (for details, see Chapter 1 in [8]), there exists a subsequence $\left\{\mu_{*}^{j_{n}}\right\}$ of $\left\{\mu_{*}^{j}\right\}$ and a probability measure $\eta_{*}$ such that $\mu_{*}^{j_{n}} \rightarrow \eta_{*}$ weakly.

Notice that $\mu_{*}^{j_{n}}$ is an optimal solution of $\mathrm{MK}^{j_{n}}$; thus, we obtain

$$
\begin{aligned}
& \Pi_{1} \mu_{*}^{j_{n}}=v_{1}^{j_{n}}, \\
& \Pi_{2} \mu_{*}^{j_{n}}=v_{2}^{j_{n}} .
\end{aligned}
$$

Using the Theorem, we have that $\Pi_{1} \mu_{*}^{j_{n}} \rightarrow v_{1}$ and $\Pi_{2} \mu_{*}^{j_{n}} \rightarrow v_{2}$ weakly. From this fact, it is clear that $\eta_{*}$ is a feasible solution of MK.

Now, we consider $\mu_{*}$ as the optimal solution of MK. from Proposition 10, we have that $\mu_{*}^{j}$ is feasible solution of $\mathrm{MK}^{j}$. It follows that

$$
\left\langle\eta_{*}^{j}, c\right\rangle \leq\left\langle\mu_{*}^{j}, c\right\rangle
$$

taking the limit when $j \rightarrow \infty$, we have

$$
\left\langle\eta_{*}, c\right\rangle \leq\left\langle\mu_{*}, c\right\rangle \text {. }
$$

On the other hand, $\eta_{*}$ is a feasible solution of MK; then

$$
\left\langle\mu_{*}, c\right\rangle \leq\left\langle\eta_{*}, c\right\rangle
$$

which completes the proof of the theorem.

\section{An Illustrative Example of This Method}

In this section, we present an example of the ideas presented in the previous section. Let $C$ be the square with vertices in the points

$$
\left\{\left(-\frac{5}{2}, \pm \frac{1}{2}\right),\left(-\frac{3}{2}, \pm \frac{1}{2}\right)\right\}
$$

and let $H$ be a hexagon with vertices being in set

$$
\left\{\left( \pm \frac{\sqrt{3}}{2}, 0\right),\left(\frac{\sqrt{3}}{4}, \pm \frac{3}{4}\right),\left(-\frac{\sqrt{3}}{4}, \pm \frac{3}{4}\right)\right\}
$$

We consider the function $c: C \times H \rightarrow \mathbb{R}$ defined by $c(x, y)=$ $\|x-y\|^{2}$. We claim to solve the following problem:

$$
\begin{aligned}
\text { MK minimize: } & \langle\mu, c\rangle \\
\text { subject to: } & \Pi_{1} \mu=\lambda_{1}, \\
& \Pi_{2} \mu=\lambda_{2}, \\
& \mu \in M^{+}(X \times Y)
\end{aligned}
$$

where $\lambda_{1}$ and $\lambda_{2}$ are the normalized Lebesgue measures to $C$ and $H$, respectively; that is, $\lambda_{1}(C)=\lambda_{2}(H)=1$. We consider the following $A B$-MRA on $\mathscr{L}^{2}\left(\mathbb{R}^{2}\right)$ :

(1) $\left\{V_{1}^{j}\right\}$, where the fundamental region is $\Delta_{1}=[0,1 / 2] \times$ $[0,1 / 2]$ and the scaling function $\varphi_{1}=2 \chi_{[0,1 / 2] \times[0,1 / 2]}$, the lattice is $\Gamma_{1}=\mathbb{Z}^{2}$, the finite group $B_{1}$ is the trivial group, and the dilation $A_{1}=\left\{2^{-j}(1,1)\right\}$.
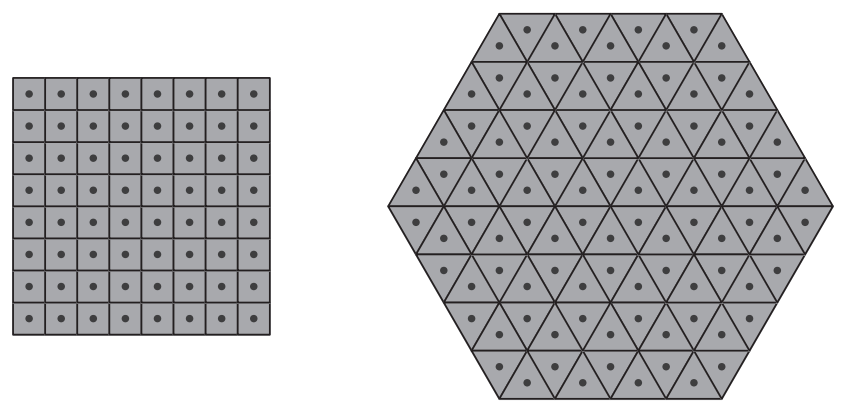

Figure 3

(2) $\left\{V_{2}^{j}\right\}$, where the scaling function $\varphi_{2}=E \chi_{\Delta_{2}}$, with $\Delta_{2}$ being the triangle with vertices in $(0,0),(\sqrt{3} / 2,0)$, and $(\sqrt{3} / 4,3 / 4)$ and $E=4 / \sqrt[4]{27}$; the lattice is $\Gamma_{2}=$ $c_{1} \mathbb{Z}^{2}$, where $c_{1}=(1 / 4)\left(\begin{array}{cc}0 & 3 \sqrt{3} \\ 6 & 3\end{array}\right)$; the finite group $B_{2}$ is the rotation group of a regular hexagon with vertices in the unit circle; the scaling is given by $A_{2}=\left\{a^{j}\right\}$, where $a=\left(\begin{array}{cc}1 & -\sqrt{3} \\ \sqrt{3} & 1\end{array}\right)$.

We denote by $C_{m}^{j}$ and $T_{n}^{j}$ the squares and the triangles associated with level $j$ of the multiresolution analysis presented above. Since $c$ is a continuous function in each pair of sets $\left(C_{m}^{j}, T_{n}^{j}\right)$, we have that $\left.c\right|_{C_{m}^{j} \times T_{n}^{j}}$ is approximated to $c\left(x_{m}^{j}, y_{n}^{j}\right)$, where $x_{m}^{j}$ and $y_{m}^{j}$ are the centroids of $C_{m}^{j}$ and $T_{n}^{j}$, respectively.

In particular, we present a discretization of $\mathrm{MK}$ problem given by (51) for the level $j=2$; the graphical description of the process of discretization is given in Figure 3 (which was generated using Mathematica 11.1.0).

Using $\left\{V_{1}^{j}\right\}$ and $\left\{V_{2}^{j}\right\}$ in $C$ and $H$, respectively, notice that $C$ is the union of congruent squares $\left\{C_{m}^{2}\right\}_{m=1}^{64}$ with disjoined interiors. Similarly, the hexagon $H$ is the union by congruent equilateral triangles $\left\{T_{n}^{2}\right\}_{n=1}^{96}$, with disjoined interiors. Note that $\lambda\left(C_{m}^{2}\right)=1 / 64$ and $\lambda\left(T_{n}^{2}\right)=1 / 96$.

In consequence, a discrete approximation for the problem defined in (51) is as follows:

$$
\begin{aligned}
\mathrm{MKD}^{2} \text { : minimize } & \sum_{m=1}^{64} \sum_{n=1}^{96}\left\|x_{m}^{2}-y_{n}^{2}\right\|^{2} \cdot \mu_{m, n}^{2} \\
\text { subject to: } & \sum_{n=1}^{96} \mu_{m, n}^{2}=\frac{1}{96} \quad m=1, \ldots, 64 \\
& \sum_{m=1}^{64} \mu_{m, n}^{2}=\frac{1}{64}, \quad n=1, \ldots, 96 \\
& \sum_{m=1}^{16} \sum_{n=1}^{24} \mu_{m, n}^{2}=1, \quad \mu_{m, n}^{2} \geq 0 \\
& \forall m=1, \ldots, 64, n=1, \ldots, 96 .
\end{aligned}
$$

The optimal solution of this linear programming problem is 4.02996. In Table 1 we present the solution for some levels. 
TABle 1

\begin{tabular}{cc}
\hline Level & Solution \\
\hline $\mathrm{MK}^{1}$ & 4.037306413676646 \\
$\mathrm{MK}^{2}$ & 4.029959684992724 \\
$\mathrm{MK}^{3}$ & 4.026748275774876 \\
\hline
\end{tabular}

[8] P. Billingsley, Convergence of Probability Measures, Wiley, New York, NY, USA, 1968.

\section{Conclusions}

The application of the Haar type multiresolution analysis (MRA) to the problem of Monge-Kantorovich allows us to obtain a discretization scheme for this problem at each level of the MRA; moreover, this MRA is based on the symmetries of the underlying space. This is an advantage because it provided us a natural method of discretization based on the geometry. Each level of the MRA induces a soluble finite linear programming problem. So, we obtain a sequence of optimal solutions of these transport problems and this sequence converges weakly to the optimal solution of the original problem.

\section{Conflicts of Interest}

The authors declare that there are no conflicts of interest regarding the publication of this article.

\section{Acknowledgments}

This work was partially supported by the CONACYT project, México. The second author is grateful to Instituto Politécnico Nacional, México, for financial support for development of this work.

\section{References}

[1] O. Hernández-Lerma and J. B. Lasserre, "Approximation schemes for infinite linear programs," SIAM Journal on Optimization, vol. 8, no. 4, pp. 973-988, 1998.

[2] J. González-Hernández, J. R. Gabriel, and O. Hernández-Lerma, "On solutions to the mass transfer problem," SIAM Journal on Optimization, vol. 17, no. 2, pp. 485-499, 2006.

[3] J. R. Gabriel, J. González-Hernández, and R. R. López-Martínez, "Numerical approximations to the mass transfer problem on compact spaces," IMA Journal of Numerical Analysis, vol. 30, no. 4, pp. 1121-1136, 2010.

[4] K. Gröchenig and W. R. Madych, "Multiresolution analysis, Haar bases, and self-similar tilings of Rn," Institute of Electrical and Electronics Engineers Transactions on Information Theory, vol. 38, no. 2, pp. 556-568, 1992.

[5] K. Guo, D. Labate, W.-Q. Lim, G. Weiss, and E. Wilson, "Wavelets with composite dilations and their MRA properties," Applied and Computational Harmonic Analysis, vol. 20, no. 2, pp. 202-236, 2006.

[6] I. A. Krishtal, B. D. Robinson, G. L. Weiss, and E. N. Wilson, "Some simple Haar-type wavelets in higher dimensions," The Journal of Geometric Analysis, vol. 17, no. 1, pp. 87-96, 2007.

[7] S. Bazaraa Mokhtar, J. John, and D. Hanif, Linear Programming and Network Flows, Wiley, 2009. 


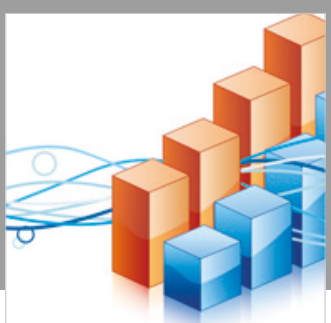

Advances in

Operations Research

\section{-n-m}
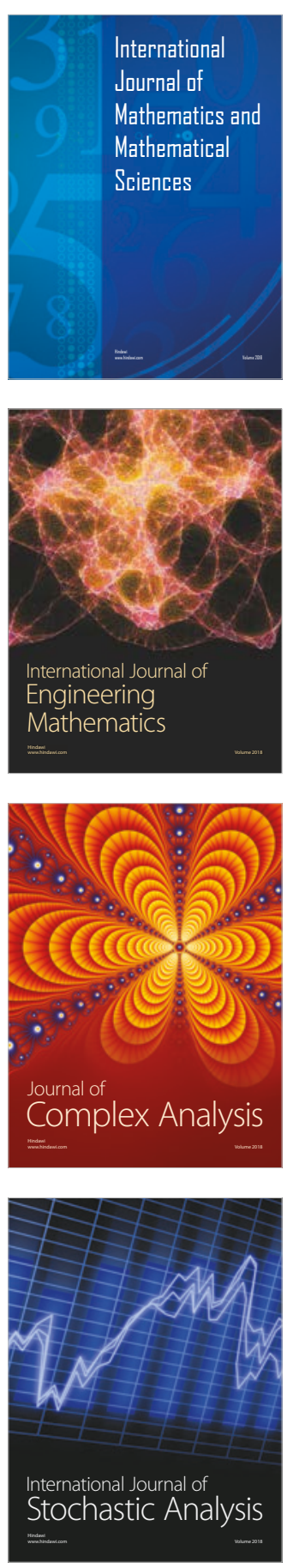
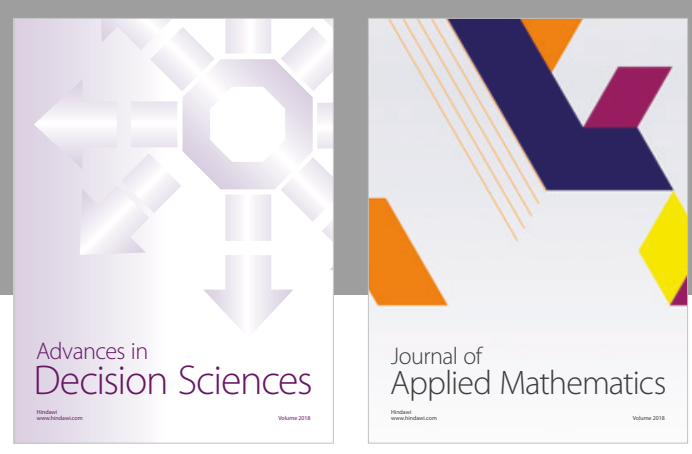

Journal of

Applied Mathematics
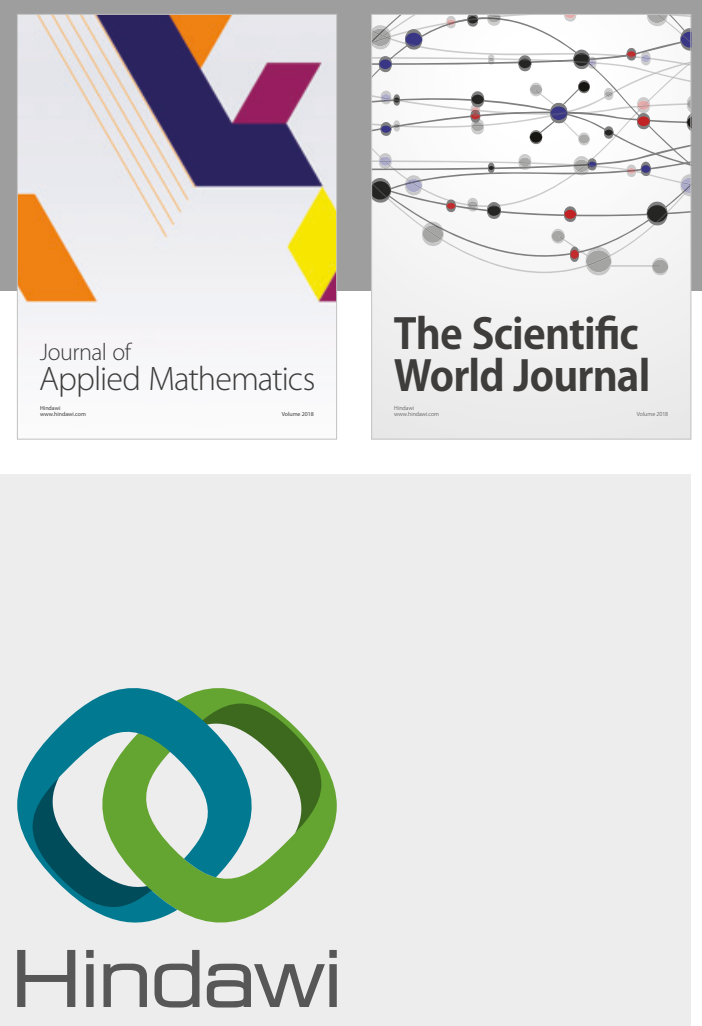

Submit your manuscripts at

www.hindawi.com

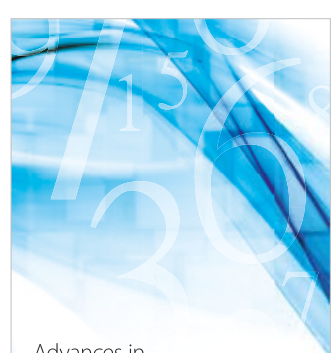

Advances in
Numerical Analysis
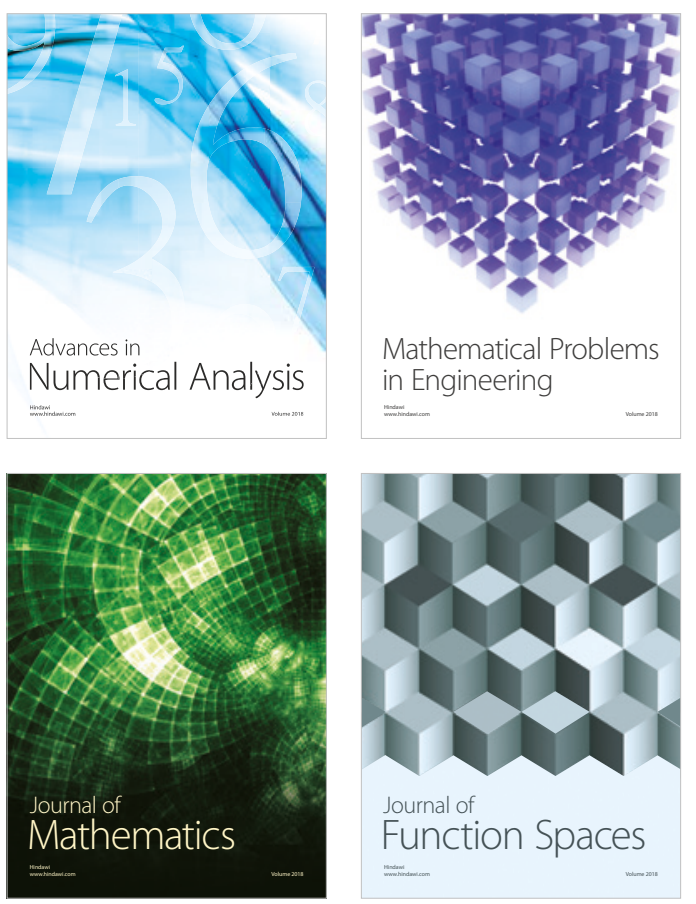

Mathematical Problems in Engineering

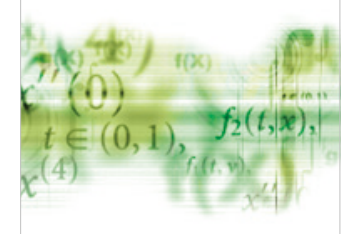

International Journal of

Differential Equations

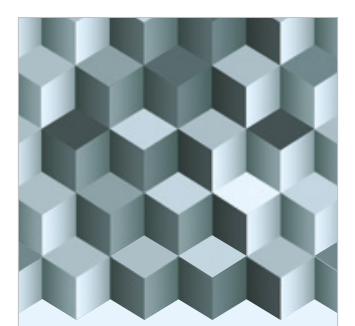

Journal of

Function Spaces

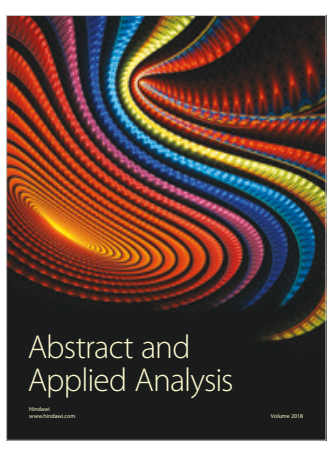

The Scientific

World Journal

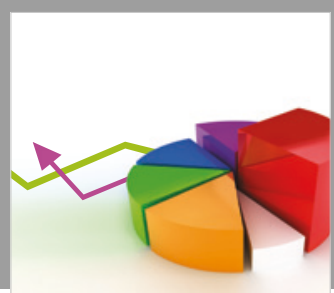

Journal of

Probability and Statistics
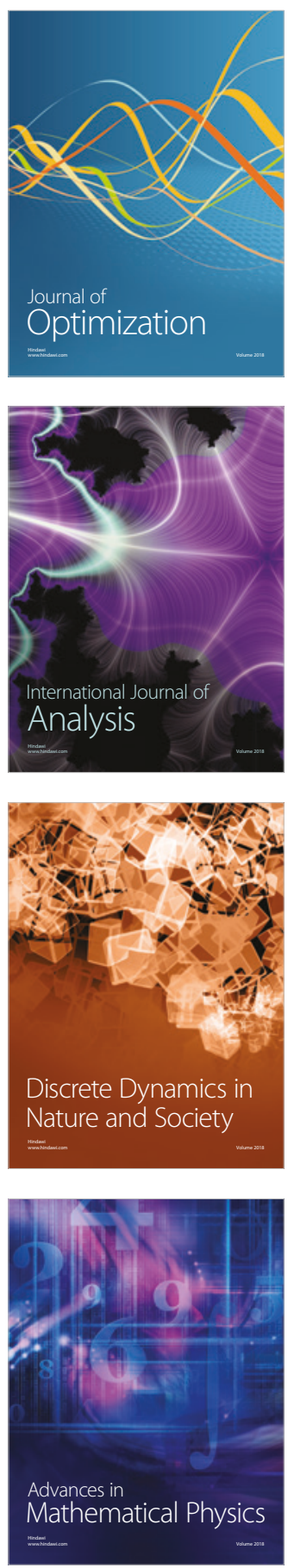